\title{
RESEARCH
}

Open Access

\section{Delayed myocardial enhancement in children with different types of cardiomyopathy: a diagnostic and prognostic tool}

Hend Gamal Mohamed Fathy Abuo Elfadl ${ }^{*}$ (D, Sabry Alam El Dean Mohamed El Mogy, Mohamed Magdy Aly Abouelkeir, Ghada Mohamad Gaballah and Nevertiti Kamal El-Din Eid

Abstract: Background: The pattern of late gadolinium enhancement (LGE) in cardiomyopathy is quite different in children compared to adults. In addition, the data about LGE imaging in children are still restricted, so the goal was to study the role of cardiac magnetic resonance (CMR) with different techniques, including LGE images in diagnosis and evaluation of different types of cardiomyopathy in children.

Results: In group A (enhancement group), LVEDV 146.2 (144) ml, indexed LVEDV 81.8 (195) ml, LVESV 50 (357) ml, indexed LVESV 47.5 (243) ml, and LVEF 36\% (64\%), and a major adverse effect was found in 12 out of 15 cases (80\%). However, in group B (non-enhancement group), the results were LVEDV 72 (303) ml, indexed LVEDV 75 (318) ml, LVESV 30 (220) ml, indexed LVESV 37.1 (189) ml, and LVEF 45.79\% (65\%), and a major adverse cardiac effect was found in 2 out of 16 cases (12.5\%). The LVEF was lower, and LV volume indices including LVEDV and LVESV were higher in patients with LGE compared to those without LGE with a statistically significant difference ( $p$ value $=$ $0.001, p$ value $=0.003$, and $p$ value $=0.005$, respectively). Furthermore, it was also found that a major adverse effect occurs with higher incidence in enhancement cases (92\%) as compared to non-enhancement cases (12.5\%) with a statistically significant difference ( $p$ value $\leq 0.001$ ). LGE was found in 15 cases out of 31 cases $(48.4 \%)$; however, the remaining 13 cases had no contrast study.

Conclusion: Cardiac MRI can be considered as an important non-invasive imaging modality, not only for assessment but also for differentiation between ischemic and non-ischemic cardiomyopathy in the pediatric age group. Using its different techniques allows a better assessment of morphologic and functional parameters in cardiomyopathy. Moreover, the late gadolinium enhancement is regarded as a promising non-invasive tool in the detection and quantification of myocardial scars. That is considered of high importance in diagnosis, categorization, and detection of etiology in most cases of different types of cardiomyopathy, in addition to risk stratification that can be an essential step in patient management.

Keywords: Cardiac magnetic resonance, Cardiomyopathy, Late gadolinium enhancement, Ejection fraction

\footnotetext{
* Correspondence: dr.hend20@yahoo.com

Faculty of Medicine, Mansoura University, Mansoura, Egypt
} 


\section{Background}

The pattern of late gadolinium enhancement (LGE) in cardiomyopathy is quite different in children compared to adults. In addition, the data about LGE imaging in children are still restricted, so the goal was to study the role of cardiac magnetic resonance (CMR) with different techniques, including LGE images in diagnosis and evaluation of different types of cardiomyopathy in children.

\section{Introduction}

Pediatric cardiomyopathies are considered a diverse group of severe illnesses of the myocardium and are responsible for significant morbidity and mortality rates in children [1]. Cardiovascular magnetic resonance imaging (CMRI) is now regarded as the gold standard imaging modality for non-invasive acquisition and processing of high-resolution temporal images for heart tissue characterization [1]. Also, delayed contrast-enhanced CMR imaging is emerging as a useful modality for detection, characterization, and differentiation of cardiomyopathies [2].

Cardiomyopathy (CM) generally refers to a diverse group of diseases of the heart muscle and are classified according to anatomy and physiology into the following types: hypertrophic cardiomyopathy (HCM), dilated cardiomyopathy (DCM), arrhythmogenic right ventricle cardiomyopathy (ARVC/D), restrictive (RCM), left ventricular non-compaction cardiomyopathy (LVNC), and unclassified cardiomyopathies. It is also classified into ischemic and non-ischemic types, depending on the presence or absence of coronary artery disease [3].

Ischemic $\mathrm{CM}$ is defined as a dysfunction of the left ventricle because of chronic lack of oxygen due to coronary artery disease. Delayed enhancement MR images show fibrosis, which appears as high signal intensity in an area of coronary artery distribution. All infarctions start sub-endocardially and may progress to transmural [4]. Non-ischemic CM has a variable etiology, i.e., genetic, toxic, metabolic, infectious, and idiopathic. In nonischemic myocardial disease, the delayed enhancement usually does not occur in a coronary artery distribution but often in mid-wall or epicardial rather than subendocardial or transmural [4].

There are other tissue characters that can be detected by MRI in non-ischemic cardiomyopathy. In primary hemochromatosis, the heart may be variably affected by increased iron deposition. Using T2*(star)-weighted imaging can detect the presence of significant amounts of iron in the heart. In some non-ischemic cardiomyopathies (e.g., myocarditis), edema may be a prominent feature and can be detected by using T2-weighted imaging [3]. Non-ischemic cardiomyopathy presents a significant diagnostic challenge, since initial symptoms are often vague and overlapping with other disease entities. Determination of the underlying cause of cardiomyopathy is essential in deciding further diagnostic and therapeutic options, and ultimately in determining prognosis [3].

Echocardiography, cardiac catheterization, and nuclear scintigraphy have limited roles in evaluation, as the appearances are non-specific [3]. End myocardial biopsy is not sensitive and hindered by sampling error. So CMR is used for accurate evaluation of myocardial morphology, function, perfusion, and tissue damage in a non-invasive way [4].

\section{Patient and method}

\section{Patient selection}

This retrospective study was carried out between January 2015 and November 2017 in the Diagnostic Radiology Department (Mansoura University Hospital). A total number of 44 patients were included in this study referred by Pediatrician cardiologists in Mansoura University children hospital for further evaluation by cardiac MRI examination. The study involved different types with nonischemic type (42 cases), including (dilated cardiomyopathy cases $(n=17), \mathrm{HCM}(n=11), \operatorname{LVNC}(n=5), \mathrm{ARVD}$ $(n=2)$, restrictive cardiomyopathy $(n=1)$, and infiltrative cardiomyopathy (6 cases)) and ischemic type (only 2 cases) as presented in Fig. 1. Thirteen patients were excluded from our study either due to contrast extravasation or parent refusal. All patients were subjected to history taking, routine laboratory investigations, 2D echocardiography, and cardiac magnetic resonance (CMR). Patients met the eligibility requirements. Patients with glomerular filtration rate $<30 \mathrm{ml} / \mathrm{min} / 1.73 \mathrm{~m}^{2}$ or frequent ventricular arrhythmias, uncontrolled diabetes mellitus, hypersensitivity to contrast media, and uncooperative children were excluded from our study. Patients with contraindications for MRI including (valve prosthesis and pacemakers that are MRI incompatible) were also excluded from the study.

Written consent was taken from all patients and this study was accepted by the IRb committee in the Faculty of Medicine, Mansoura University.

\section{Cardiac magnetic resonance}

CMR imaging was performed with a magnetic resonance scanner (Ingenia 1.5 T; Philips, Medical Systems, Best, the Netherlands). A 16-channel torso phased-array receiver coil was used for signal reception. All data acquisition was retrospective ECG gated. Patients were examined in supine position. Children ( $>7$ years) who could control breathing data were acquired with respiratory gating. Small children ( $<7$ years) were sedated with $10 \%$ chloral hydrate and examined under free-breathing condition. 


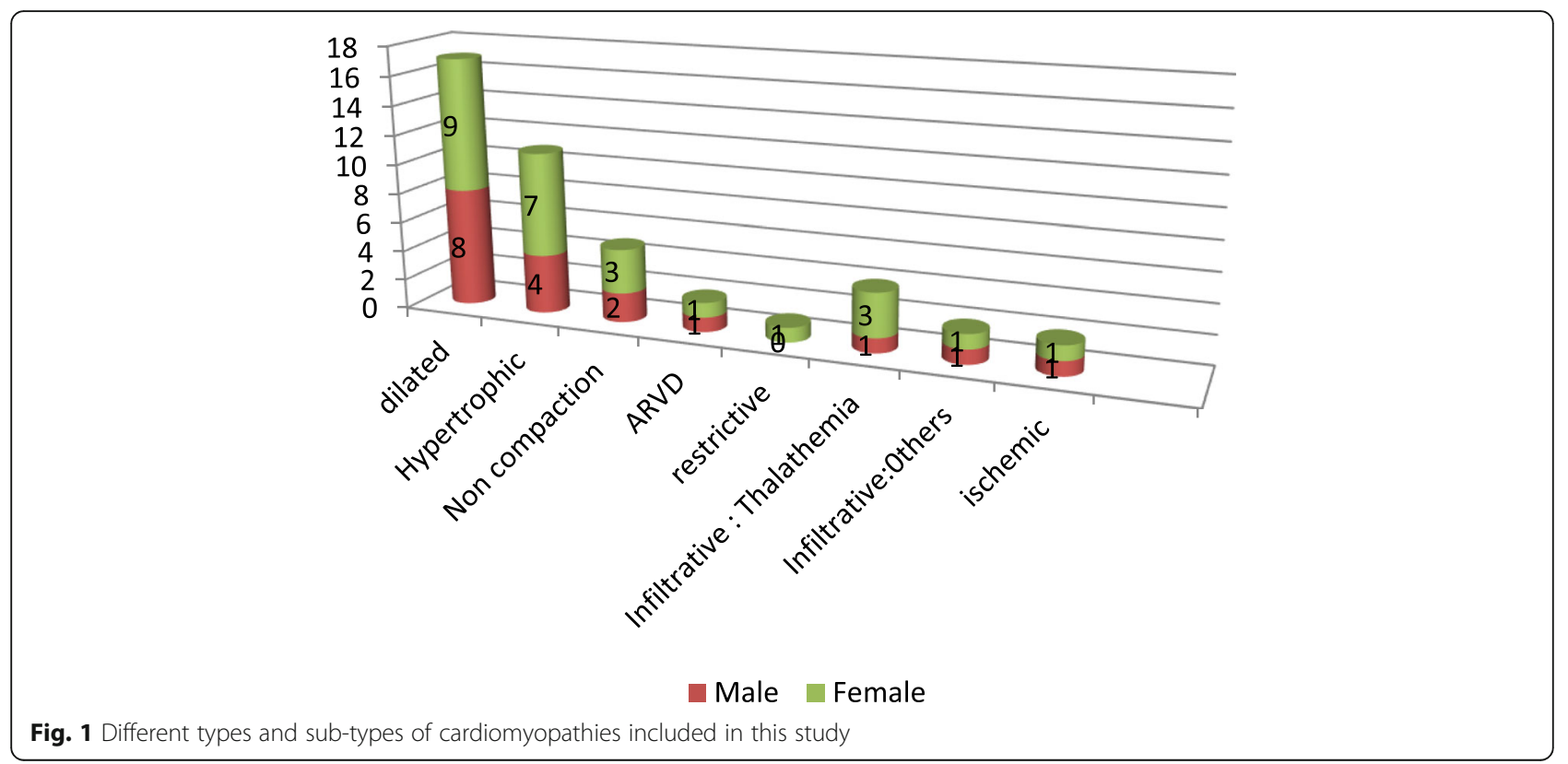

\section{CMRI acquisition protocol}

The scan protocol was carried out in the following order: Scout images (axial, coronal, sagittal) using realtime interactive planning (FOV $450 \times 450 \mathrm{~mm}^{2}$ ), slice thickness $10 \mathrm{~mm}$, acquisition matrix $220 \times 176$, voxel size $1.6 \mathrm{~mm} \times 1.9 \mathrm{~mm} \times 10 \mathrm{~mm}$, echo/repetition time (TE/TR) shortest, and flip angle $50^{\circ}$. Cine steady-state free precession (SSFP) sequences acquired on the longaxis cardiac planes: four chambers, two chambers, and three chambers (FOV $350 \times 350 \mathrm{~mm}^{2}$, slice thickness $6-$ $8 \mathrm{~mm}$, acquisition matrix $220 \times 176$, voxel size $1.6 \mathrm{~mm} \times$ $1.9 \mathrm{~mm} \times 10 \mathrm{~mm}$, echo/repetition time (TR/TE) $6.1 / 3 \mathrm{~ms}$ and flip angle $60^{\circ}$ ), followed by a "stack" of contiguous SSFP cine images, with the same technical parameters, acquired along cardiac short axis, to cover the whole ventricle from base to apex. T2-weighted images acquired in 4-chamber and SA with a triple-inversion recovery fast spin echo sequence to evaluate myocardial edema. Imaging parameters were TR, $1500 \mathrm{~ms}$; TE, 31 ms; FA, 90; FOV, 350-350 $\mathrm{mm}^{2}$; matrix, 196-149; and slice thickness, $8 \mathrm{~mm}$. After $10-15 \mathrm{~min}$ of intravenous administration of contrast agent (DOTAREM, Magnevest), at $0.2 \mathrm{mmol} / \mathrm{kg}$, T1-weighted inversion recovery sequences (PSIR-TFE-BH) (FOV $350 \times 350 \mathrm{~mm}^{2}$, slice thickness $8-10 \mathrm{~mm}$, acquisition matrix $220 \times 176$, voxel size $1.6 \times 1.9 \times 10, \mathrm{TR} / \mathrm{TE} 6.1 \mathrm{~ms} / 3 \mathrm{~ms}$, flip angle $25^{\circ}$, and inversion time (TI) $250-425 \mathrm{~ms}$ ) were acquired along the same planes of the cine SSFP images, in order to evaluate the presence of LGE. To determine the correct TI, for nulling normal myocardial signal intensity, a Look-Locker sequence was acquired in one short-axis plane. Steady-state free precession cine images and $\mathrm{T} 1$ inversion recovery sequence for determining LGE, both were acquired in breath hold.

\section{Left ventricular systolic function}

The cine SSFP acquisitions acquired in short axis were transferred to workstation (Philips extended work station (EWS)), after the manual tracing of the endocardial and epicardial contours of the left ventricular wall at end diastole and end systole on the short axis cine images, LV global function was calculated. End-diastolic volume (EDV), end-systolic volume (ESV), and ejection fraction (EF) were calculated for each patient. Subsequently, both EDV and ESV have been indexed for body surface area (BSA). Wall thickness and wall motion abnormality were also assessed.

\section{Analysis and interpretation of late gadolinium enhancement}

Segmental enhancement was quantified by the Philips extended workstation (EWS). View in all 17 segments according to the American Heart Association (AHA) classification. The presence of enhancement in each segment was scored by visually assessing the area showing late gadolinium enhancement as compared with the normal LV myocardium in each segment. Images were evaluated for the presence, distribution, pattern, and site of delayed myocardial enhancement.

\section{Echocardiographic assessment}

Transthoracic echocardiography was performed by Phillips ultrasound machine with a transducer of 2-4 MHz. Routine short axis, 4- and 2-chamber views were obtained in two to three cardiac cycles for the assessment of ejection 
fraction, stroke volume, ventricular and atrial dimensions, wall thickness, segmental wall motion at apex, mid cavity, and base of both ventricle. Measurements were calculated by modified Simpson's rule.

\section{Statistical analysis}

Data entry and statistical analyses were performed using SPSS (statistical Package of Social Sciences) version 16.0 (SPSS Inc., Chicago, IL, USA). The appropriate statistical tests were used. Parametric data were expressed in mean \pm standard deviation. Non-parametric data were expressed in median, minimum, and maximum. The normality of data was first tested by one-sample K-S test. In addition, independent $t$ test was used to compare means for continuous parametric variables of each two different groups. In addition, Mann-Whitney $U$ test (z) was used to compare non-parametric continuous variables in two different groups. Pearson Chi-square tests were used to compare the categorical variables between both cases and control groups. $p$ value $<0.05$ was considered as statistically significant.

\section{Results}

Forty-four (44) patients were included in this study. The mean age was $9.8 \pm 5.6(2-18)$ years. Main demographic data of the overall population are resumed in Table 1 . Within the whole population, the mean LVEF was LV end-diastolic indexed volume and other main CMR findings are summarized in Table 2. The study population was divided into two groups: patients with the presence of LGE (group A, 15 patients) and patients with no evidence of significant LGE (Group B, 16 patients) (Tables 3 and 4). The two subgroups were matched for age and sex. In group A (enhancement group), the results were expressed as median (range): LVEDV 146.2 (144) ml, indexed LVEDV 81.8 (195) ml, LVESV 50 (357) ml, indexed LVESV 47.5 (243) ml, and LVEF 36\% (64\%), and

Table 1 Demographic data of studied group (44 children)

\begin{tabular}{lll}
\hline & Mean SD (min-max) & \\
\hline Age/years & $9.8 \pm 5.6(2-18)$ & \\
Weight (in kg) & $38.1 \pm 23(8-80)$ & \\
Height (in cm) & $124.2 \pm 37.4(60-178)$ & \\
BSA & $1.12 \pm 0.51(0.42-1.98)$ & \\
Sex & $N$ & $\%$ \\
$\quad$ Male & 18 & 40.9 \\
$\quad$ Female & 26 & 59.1 \\
Family history & 5 & 11.4 \\
Consanguinity & 5 & 11.4 \\
Major adverse effects* & 14 & 31.8 \\
\hline
\end{tabular}

*Major adverse effects include hospitalization for HF (five cases), death (five cases), arrhythmia (three cases), and brain infarction (1 case) a major adverse effect was found in 12 out of 15 cases (80\%). However, in group B (non-enhancement group), the results were LVEDV 72 (303) $\mathrm{ml}$, indexed LVEDV 75 (318) ml, LVESV 30 (220) $\mathrm{ml}$, indexed LVESV 37.1 (189) $\mathrm{ml}$, and LVEF $45.79 \%$ (65\%), and a major adverse cardiac effect was found in 2 out of 16 cases (12.5\%). The LVEF was lower, and LV volume indices including LVEDV and LVESV were higher in patients with LGE compared to those without LGE with a statistically significant difference ( $p$ value $=0.001, p$ value $=0.003$, and $p$ value $=0.005$, respectively) as presented in Table 5 . Furthermore, it was also found that a major adverse effect occurs with higher incidence in enhancement cases (92\%) as compared to non-enhancement cases (12.5\%) with a statistically significant difference $(p$ value $\leq 0.001)$ as presented in Table 6 . As regards the delayed contrast enhancement finding in the study, LGE was found in 15 cases out of 31 cases (48.4\%) (Table 3). However, the remaining 13 cases had no contrast study. The cases with LGE were 13 non-ischemic cases (seven dilated cardiomyopathy, 4 hypertrophic cardiomyopathy, 1 arrhythmogenic right ventricular dysplasia (case number 5), and one infiltrative) and two ischemic cases. With the following regional patterns (Table 4): mid-wall ( $n=9$ cases) as present in Fig. 4 sub-endocardial extending to epicardial surface (transmural) ( $n=4$ cases) as presented in Fig. 3, sub-epicardial $(n=2$ cases $)$, and sub-endocardial $(n=2$ cases) as presented in Fig. 3, there was overlapping in total number as two cases show both mid-wall and epicardial enhancement. Bland-Altman plot test for agreement between MRI and echocardiography for measurement of EF was done as presented in Fig. 2.

\section{Discussion}

Pediatric cardiomyopathies caused by different causes either ischemic or non-ischemic and primary (genetic) or secondary (non-genetic). They have diagnostic challenge to clinicians, so it is important to detect the underlying cause as it then leads to improvement of outcome with disease-specific treatment [5].

As reported by Cox et al. [6], the patient population of pediatric cardiomyopathies was divided into 4 types: dilated $(53.8 \%)$, hypertrophic (34.2\%), restrictive (3.2\%), and other or mixed $(8.9 \%)$. And these findings are in approximation with result of our study in which DCM represents $43.1 \%$ and was the most common form followed by hypertrophic (25\%) and restrictive (2.3\%).

Nineteen out of $44(43.1 \%)$ cases were dilated cardiomyopathy. These patients were retrospectively assessed and most of these cases have an idiopathic form (42\%) and this agrees with the study conducted by Hong [6], who reported that in pediatric population most cases have idiopathic forms of disease and maybe, in the future, these cases will be found to have genetic or familial 
Table 2 Left ventricle parameters of CMR in studied children of different sub-types of cardiomyopathy

\begin{tabular}{|c|c|c|c|c|c|}
\hline Sub-types of cardiomyopathies & $\begin{array}{l}\text { LVEF\% median } \\
\text { (range) }\end{array}$ & $\begin{array}{l}\text { LVEDV (ml) } \\
\text { median (range) }\end{array}$ & $\begin{array}{l}\text { LVEDVI median } \\
\text { (range) }\end{array}$ & $\begin{array}{l}\text { LVESV (ml) } \\
\text { median (range) }\end{array}$ & $\begin{array}{l}\text { LVESVI median } \\
\text { (range) }\end{array}$ \\
\hline Primary dilated & $19.7(12-54)^{b, c, d}$ & $208.8(76-412)^{b, c, d}$ & $170.1(89-338)^{a, b, c, d}$ & $162.9(53-362)^{b, c, d}$ & $125.5(41-257)^{b, c, d}$ \\
\hline Secondary dilated post myocarditis & $29.5(9-72)^{\mathrm{e}}$ & $157(121-367)^{e, f}$ & $82.6(70-195)^{a, e}$ & $101.5(42-327)^{\mathrm{e}}$ & $56.7(23-174)^{e}$ \\
\hline Secondary dilated toxins, drugs & $43.8(32-56)$ & $43.4(32-55)$ & $41(23-59)$ & $22.8(22-24)$ & $20.7(16-26)$ \\
\hline Hypertrophic & $65(45-81)^{b, e}$ & $51(16-172)^{b, e}$ & $75(21-117)^{b, e}$ & $26.4(6-59)$ b, e & $26.3(12-45)^{b, e}$ \\
\hline Non-compaction & $60(6-67)^{c}$ & $63(6-135)^{c, f}$ & $66.3(15-250)^{c}$ & $24(3-127)^{c}$ & $56.7(6-235)^{c}$ \\
\hline ARVD & $42.3(53-63)$ & $68.3(53-84)$ & $73.8(55-92)$ & $30(29-32)$ & $32.3(30-35)$ \\
\hline Restrictive & * & * & * & * & * \\
\hline Infiltrative thalassemia & $43.5(21-66)^{d}$ & $113.8(16-128)^{d}$ & $110.7(18-119)^{d}$ & $66(5-96)^{d}$ & $62.3(6-94)^{d}$ \\
\hline Infiltrative others & $70.2(69-72)^{d}$ & $79.5(26-134)^{d}$ & $63.6(56-71)^{d}$ & $23(8-38)^{d}$ & $18.9(18-20)^{d}$ \\
\hline Ischemic & $40.5(35-46)$ & $137.5(19-256)$ & $86.6(41-132)$ & $75.2(12-138)$ & $49(27-71)$ \\
\hline Test of significance & 24.7 & 21.7 & 22.2 & 24.9 & 22.3 \\
\hline$p$ value & $0.006^{*}$ & $0.016^{*}$ & $0.014^{*}$ & $0.005^{*}$ & $0.013^{*}$ \\
\hline
\end{tabular}

Similar letters indicate significant difference

causes with subsequent changes in the epidemiology of disease [7].

However, Latus et al. [8] reported that majority of the cases in pediatric population have idiopathic form, the most common known causes are myocarditis (46\%) and this with our study results in which among studied dilated cases, we found that myocarditis is the commonest cause of dilation found in seven patients (41\%) (Fig. 3).

In our study, the cardiac MRI was highly effective in the assessment of ventricular volumes with increased indexed EDV of the left ventricle, reduced EF and global hypokinesia in all cases (100\%) of DCM. The left ventricular wall thinning presents only in advanced cases. One of the cases has associated dilated right ventricle. All our previous results in DCM are in agreement with all previous study in literatures by Iacucci et al. [7] and Lehrke et al. [9].

Eleven out of 42 cases (26.2\%) in our study, patients were diagnosed as hypertrophic cardiomyopathy (HCM) and represent the second common type in our study, this finding is a close match with a previous study reported by Hong [6] as mentioned before.

In all our cases, diagnosis based on both echo and MRI findings with mean thickness were $13 \mathrm{~mm}$. Ten out of 11 cases showing asymmetric focal left ventricular hypertrophy and only one case with apical form. These findings are in close match with a previous study conducted by Rickers et al. [10], they reported that asymmetric involvement of the inter-ventricular septum is the most common form of the disease, accounting for an

Table 3 LGE result in study groups (after exclusion of non-contrast study)

\begin{tabular}{|c|c|c|}
\hline Types and sub-types of cardiomyopathies & $\begin{array}{l}\text { No enhancement } \\
(N=16)\end{array}$ & $\begin{array}{l}\text { Enhancement } \\
(N=15)\end{array}$ \\
\hline Primary dilated & $5(35.3)$ & $1(6.6)$ \\
\hline Secondary dilated post myocarditis & - & $6(40)$ \\
\hline Secondary dilated toxins, drugs & $1(5.9)$ & - \\
\hline Hypertrophic & $10(58.8)$ & $4(26.6)$ \\
\hline Non-compaction & - & - \\
\hline ARVD & - & $1(6.6)$ \\
\hline Restrictive & - & - \\
\hline Infiltrative thalassemia & - & - \\
\hline Infiltrative others & - & $1(6.6)$ \\
\hline Ischemic & - & $2(13.3)$ \\
\hline Test of significance & 19.4 & \\
\hline$p$ value & $0.002^{*}$ & \\
\hline
\end{tabular}

*Thirteen cases were excluded from study either due to escape of contrast out of cannula or due to no administration of contrast 
Table 4 Pattern of LGE in study groups

\begin{tabular}{llll}
\hline Sub-types of cardiomyopathies & Pattern of enhancement & & \\
\cline { 2 - 4 } & Sub-endocardial & Mid-wall & Sub-epicardial \\
\hline Primary dilated & - & 1 & $2^{*}$ \\
Secondary dilated post myocarditis & - & $2^{*}$ & - \\
Secondary dilated toxins, drugs** & - & - & - \\
Hypertrophic & - & 4 & - \\
Non-compaction & - & - & - \\
ARVD & - & $1^{* * *}$ & - \\
Restrictive & - & - & - \\
Infiltrative thalassemia & - & - & - \\
Infiltrative others & - & 1 & - \\
Ischemic & 2 & - & - \\
\hline
\end{tabular}

*There is overlapping in the total number as two cases show both the mid-wall and epicardial enhancement

**No LGE; however, there is increase first pass perfusion

***The enhancement involving RVOT

estimated $60 \%$ to $70 \%$ of the cases of $\mathrm{HCM}$ and this is in approximation of our finding (90\%) in our HCM cases, and these relatively high percentage in our study may be due to our small sample size.

Also, Rickers et al. [10] reported that hypertrophy in this phenotype is typically most evident in the antroseptal myocardium and this is a close match with our study in which asymmetric hypertrophy adjacent to the left ventricular outflow tract including the anterior and infero-septal walls present in more than two thirds (91\%) of our patients. However, areas of relative sparing of hypertrophy are also commonly visualized in HCM patients but this finding is not noted in our study.

Systolic anterior motion (SAM) effect is found in 6 out of 10 cases $(60 \%)$ of focal sigmoid-shaped hypertrophy and this matched with the study that records the presence of SAM in $31-61 \%$ of HCM in his study and it is associated with resting LVOTO in $25-50 \%$ of cases

Table 5 Comparison between demographic and functional MRI findings of all studied groups between enhancement and nonenhancement groups using Mann-Whitney test

\begin{tabular}{llll}
\hline & $\begin{array}{l}\text { Enhancement } \\
(n=15)\end{array}$ & $\begin{array}{l}\text { No enhancement } \\
(n=16)\end{array}$ & $p$ value \\
\hline $\begin{array}{l}\text { Age (year) median (Range) } \\
\begin{array}{l}\text { Weight (in kg) median } \\
\text { (range) }\end{array}\end{array}$ & $16(16)$ & $10(15)$ & 0.068 \\
$\begin{array}{l}\text { Height (in cm) median } \\
\text { (range) }\end{array}$ & $125(172)$ & $169(66)$ & 0.05 \\
BSA median (range) & $1.79(1.51)$ & $0.93(0.47)$ & 0.05 \\
LVEDV (ml) median (rang) & $146.2(144)$ & $72(303)$ & 0.06 \\
LVEDVI median (pange) & $81.8(195)$ & $75(318)$ & $0.003^{*}$ \\
LVESV (ml) median (range) & $50(357)$ & $30(220)$ & $0.007^{*}$ \\
LVESVI median (range) & $47.5(243)$ & $37.1(189)$ & $0.005^{*}$ \\
LVEF \% median (range) & $36(64)$ & $45.79(65)$ & $0.003^{*}$ \\
\hline
\end{tabular}

(based on several series studies) [11-13]. And this matched with our finding in which the turbulent jet through the left ventricular outflow track coupled with an eccentric jet of mitral regurgitation, that was highly suggestive of a significant LVOT obstruction which was noted in 7 of our cases (63.6\%) (Fig. 4).

Three out of these cases with LVOT obstruction showed papillary muscle abnormalities in the form of accessory papillary muscle, bifid muscle with abnormal insertion in to mitral valve leaflet and all three cases showing LVOT obstruction, and these findings are with a previous reported study by Maron and Maron [14] that found resting or provo cable LVOT obstruction in up to $70 \%$ of HCM cases and relates it to complex anatomical relationships between the basal septum, LVOT, mitral valve, and papillary muscles (Fig. 4).

Three out of our $11 \mathrm{HCM}$ cases (27.2\%) had family history of HCM. And this is a close match with the study conducted by Maron et al. [15] that found eleven (27.5\%) of his patients had a family history of HCM at the time of his study. We assessed two cases (sisters to already diagnosed one of study cases) without HCM diagnosis or any clinical suspicion as trial for MRI screening, and LV crypt was seen in two cases together with mild focal asymmetrical hypertrophy in anteroseptal segment.

Table 6 Comparison between the percentage of major adverse effect between enhancement and non-enhancement groups in study

\begin{tabular}{llll}
\hline & $\begin{array}{l}\text { Non-enhancement } \\
\text { Major adverse effect }\end{array}$ & Enhancement & $p$ value \\
\hline No & $14(87.5 \%)$ & $3(20 \%)$ & ${ }^{*} p \leq 0.001$ \\
Yes & $2(12.5 \%)$ & $12(80 \%)$ & \\
\hline
\end{tabular}




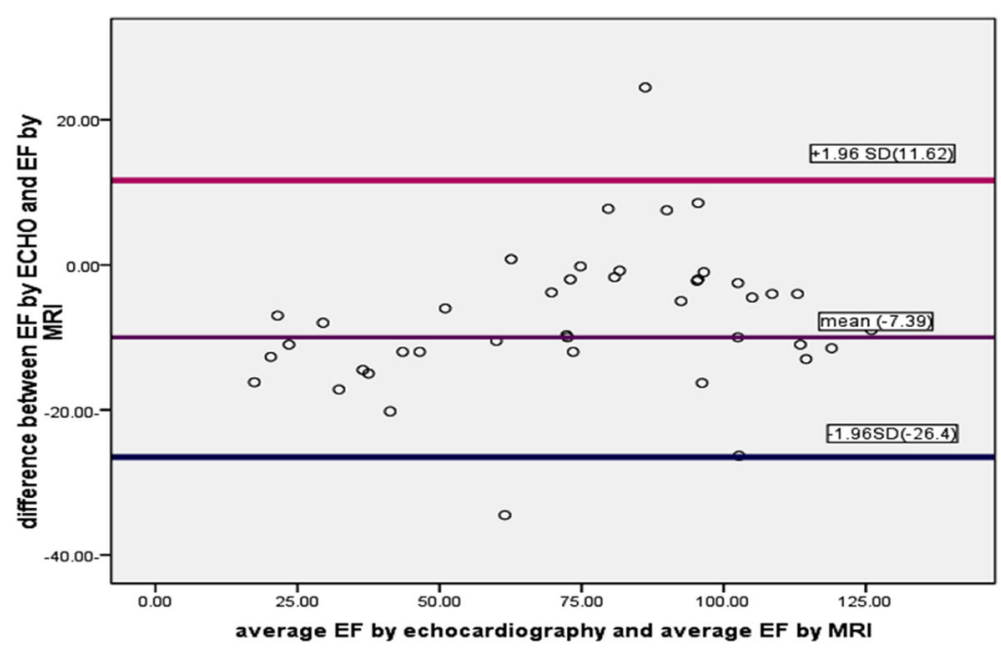

Fig. 2 Bland-Altman plot test for agreement between MRI and echocardiography for measurement of EF

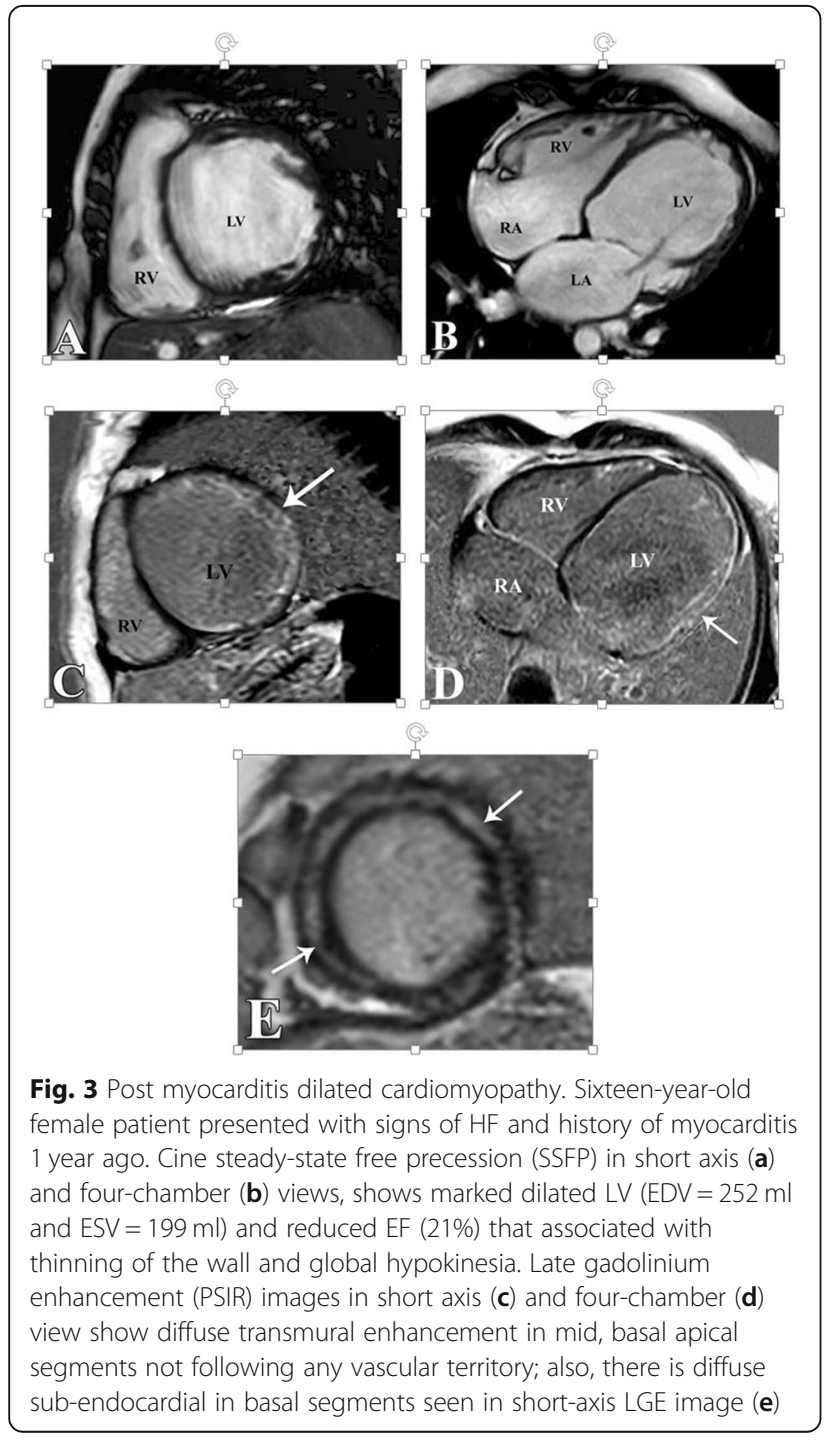

However, we only represent only two cases but this matched with other previously mentioned results by Moon and McKenna [16] that documented screening of the family members of a patient with HCM is important because the first-degree relatives of such a patient have a $50 \%$ chance of being a gene carrier; he found a high rate of occurrence of $81 \%$ (13 of 16 subjects) in cine MR imaging in HCM mutation carriers who had not yet developed LV hypertrophy. And this may be a point for further research study as a non-invasive technique for screening.

We have diagnosed LVNC in only five cases out of referral eight cases (previously diagnosed by echo) (62.5\%). We depend on MRI diagnostic criteria published by a study conducted by Mikolich et al. [17] in which they found 15 of 22 patients (68\%) had a diagnosis of $\mathrm{LVH}$ on their 2-D echo study, while only 3 had LVH on their cardiac MRI study. Based on these data, patients with LVH on 2-D echo should be considered for a cardiac MRI examination to determine if the increased LV wall thicknesses are truly LVH or an undiagnosed LV noncompaction.

Zuccarino et al. [18] reported that the apical and mid ventricular segments of both the inferior and lateral walls are the most common affected segments in LVNC cases and this with our study results in which all cases had shown region of non-compaction along the lateral wall and sparing septal wall (Fig. 5).

Three of our patient have associated other congenital heart disease, one case has hypoplastic left heart syndrome while another one has PDA and the other ASD and this matches with study conducted by Ichida [19] who described this as a non-isolated form. The remaining two cases have an isolated form and this was 


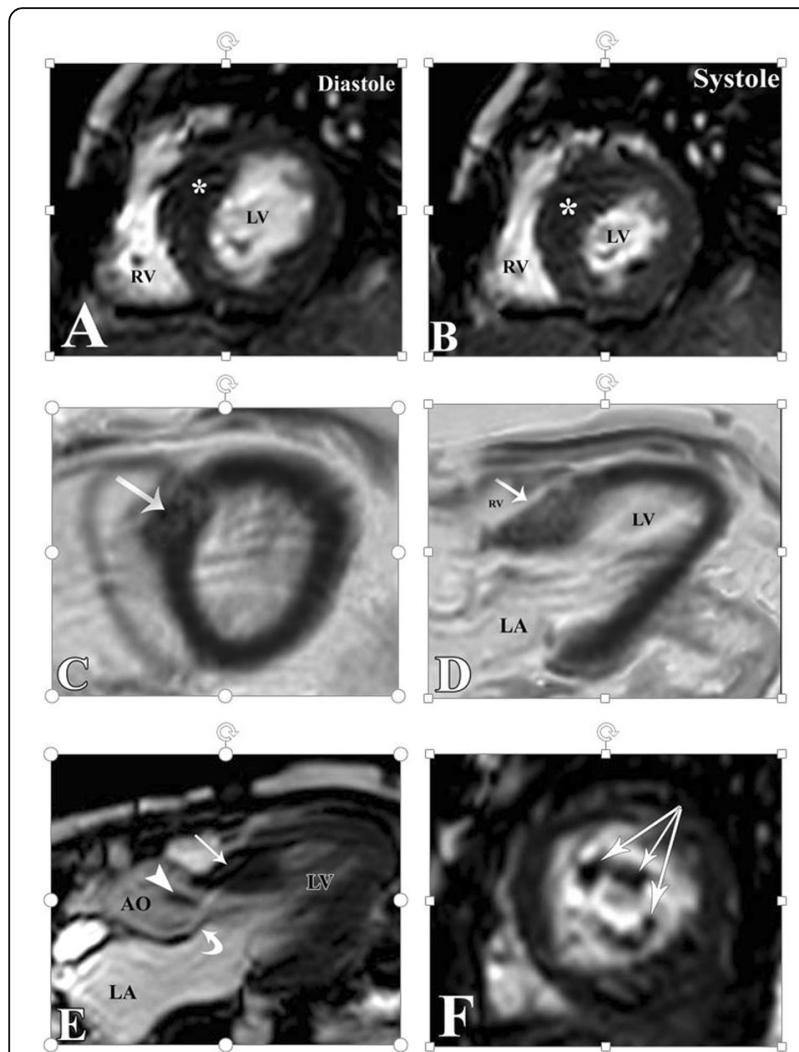

Fig. 4 a-f Residual HCOM with LVOT obstruction by systolic anterior motion of the mitral valve (SAM) and anomalous papillary muscle: Nine-year-old male patient presented with status post myoctomy with residual HOCM. Cine steady-state free precession (SSFP) in short axis $(\mathbf{a}, \mathbf{b})$ views, shows residual focal asymmetrical hypertrophy of antero-septal segment of left ventricle (site of previous myomectomy) with maximum thickness about $20 \mathrm{~mm}$ in end diastole image (a) and end systole image (b) with hyper dynamic EF $(73 \%)$ normal both $(E D V=86.4 \mathrm{ml}$ and $E S V=35.7 \mathrm{ml})$. Three-chamber view (e) in the same patient shows thickening of the basal ventricular septum causing LVOT narrowing. LV contraction leads to flow acceleration in the narrowed LVOT with visible area of signa void (arrow, e) with subsequent pressure drops cause SAM and leading to mitral regurgitation (MR) (black arrow, e). f Short axis plane in the same patient showing the extra (anomalous) papillary muscle

different from the study conducted by Zuccarino et al. [18] who reported that the isolated form is the commonest form.

The incidence of ARVD in our study was (4.7\%); however, the true incidence of ARVD is unknown and is categorized as a rare disease with estimated prevalence at 1 : 5000 [2]. We have two cases with ARVD diagnosis. One of them showed a normal-size right ventricle with reduced ejection fraction (30\%), regional hypokinesia and dyskinesia with small aneurysmal dilation near RVOT, and no detected fatty infiltration in the right or left ventricle; also, this case demonstrated right atrial dilation secondary to tricuspid regurge; there was subtle patchy enhancement in RVOT. The other case shows fatty infiltration was detected in RVOT with dilated RV and RA with tricuspid regurgitation; however, no detected WMA (Fig. 6).

In a study carried out by Grosse-Wortmann et al. [20], they found that MRI is a useful tool in the diagnostic work-up of ARVC in children and adolescents in contrast to adult due to more subtle degree of WMA in this age group which are not detected by echocardiography but found on MRI. Also, in the pediatric age group, fibrofatty degeneration is found rarely and never without wall motion abnormalities, and this matches with the finding in our two cases.

As regards restrictive cardiomyopathy type, in our study, we have six referral patients with diastolic dysfunction; one of them has restrictive cardiomyopathy and the other five cases are diagnosed as constrictive pericarditis based on clinical, echo, and MRI findings. Although both restrictive cardiomyopathy and constrictive pericarditis are characterized by diastolic dysfunction and impairment of ventricular filling, they have different management and differentiation between them is very important.

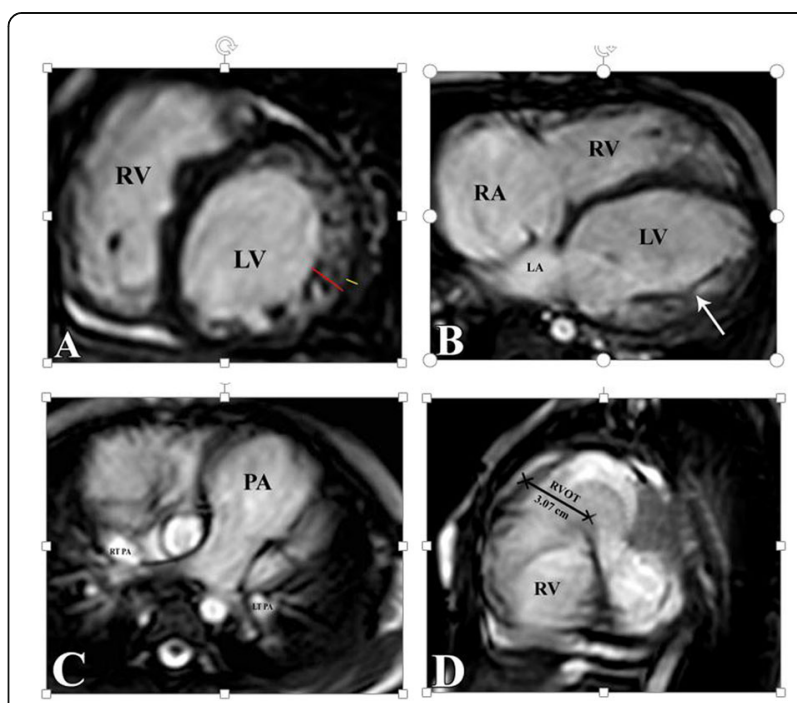

Fig. 5 a-d LVNC and picture of pulmonary hypertension. Threeyear-old male patient presented with signs of pulmonary hypertension. Cine steady-state free precession (SSFP) in short axis and four-chamber cine images $(\mathbf{a}, \mathbf{b})$. Short axis image in diastole with red line denotes the thickness of the non-compacted myocardium, yellow line denotes the compacted myocardium and prominent trabecular network along the mid anterior, lateral, and inferior left ventricular wall segments with maximum thickness of non-compacted to compacted area in end diastole $=3.6$ correlates with LVNC criteria (normal reference $>2.3$ ). Both images $(\mathbf{a}, \mathbf{b})$ show dilated left ventricle with global hypokinesia, reduced EF (6.2\%). There is marked dilatation of right ventricle which shows reduced ejection fraction (8.2\%) associated with global hypokinesia and prominent trabeculation. There is dilated RVOT, pulmonary artery, and its two branches $(\mathbf{c}, \mathbf{d})$ 
In restrictive case, there were small both ventricles with significant decrease in EDV and ESV; however, there were normal EF of both ventricle and marked dilation of both atria associated with dilated IVC and hepatic veins while no pericardial thickening or effusion or pericardial enhancement (Fig. 7).

While in the other five cases that were diagnosed as constrictive pericarditis, there were also small both ventricles with significant decrease in EDV and ESV and normal EF of both ventricle; however, the main differentiating points were thickened pericardium with no enhancement in chronic cases and enhancement in acute setting and evidence of septal bounce that is more evident with respiration, flattening of the inter-ventricular septum with inspiration, and septum bounces to the right side with expiration.

In all studied referral cases, both morphological and functional parameter of restrictive cardiomyopathy and constrictive cardiomyopathy are similar apart from septal bounce which is diagnostic for constrictive pericarditis and detected on MRI using a real-time imaging sequence in the short-axis plan and thickened enhancing or non-enhancing pericardium and this point is superior assessed by MRI than echo. Our findings are a closer match with study conducted by Rammos et al. [21] that use the respiratory-related ventricular coupling to differentiate patients with constrictive pericarditis and RCM.

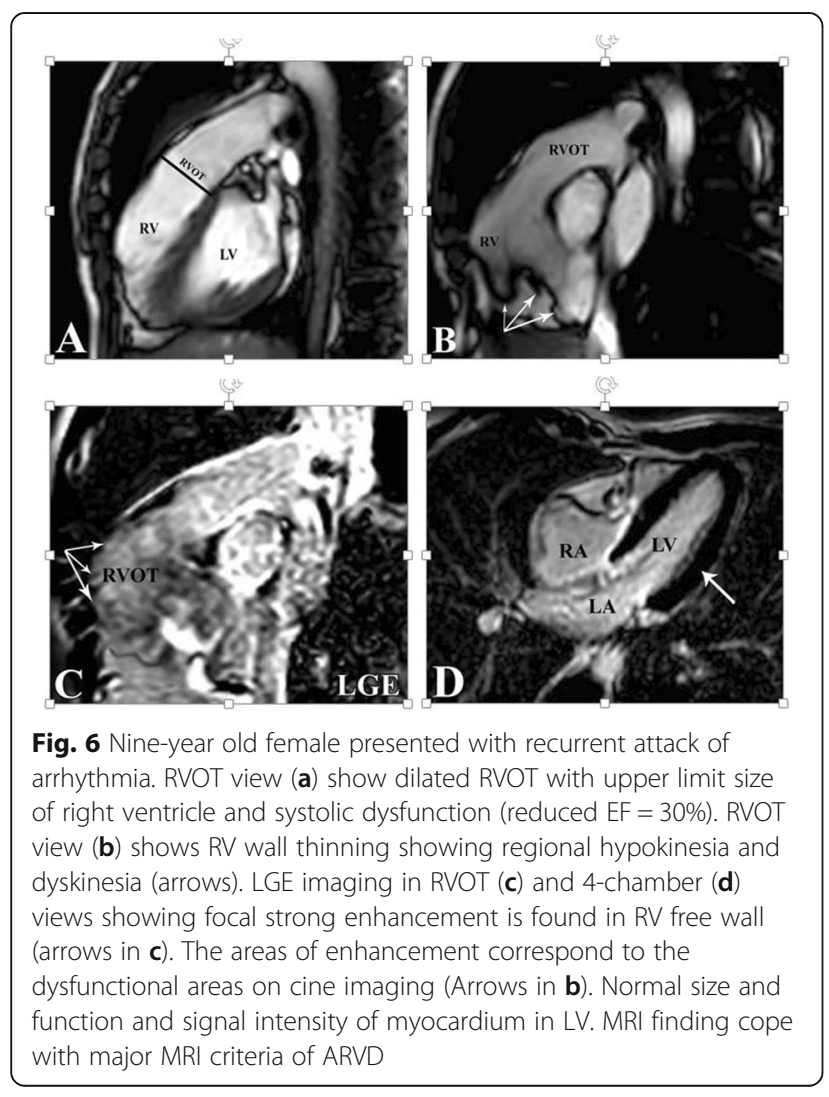

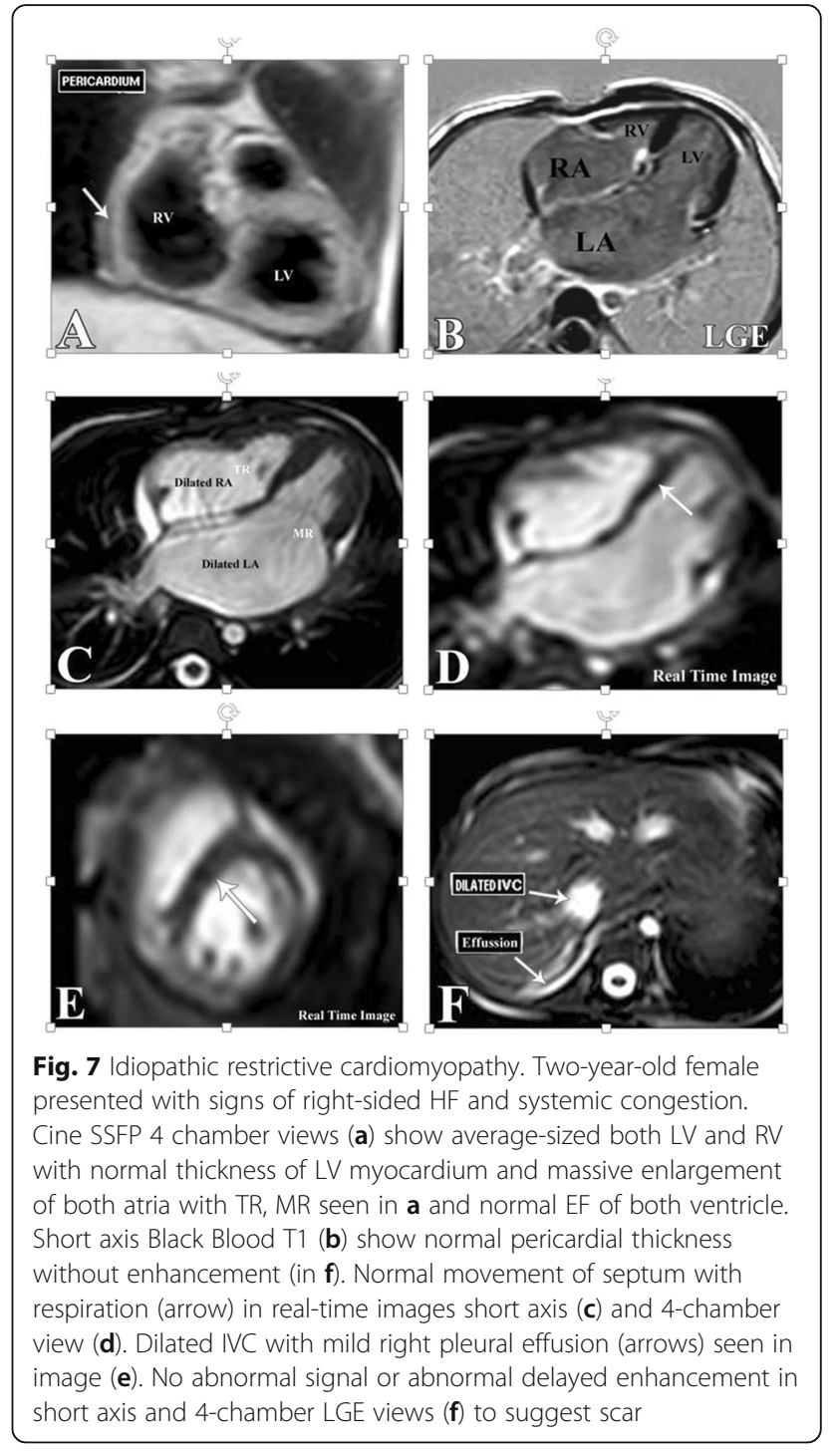

However, the cases of constrictive pericarditis were excluded from our study and this is the only differentiating point.

Another form of restrictive cardiomyopathy type is the infiltrative form which also enters under secondary causes as previously mentioned in review. In our study, we have six cases; two of them with metabolic storage disease and were presented with diffuse hypertrophy as differential diagnosis to HCM cases while the remaining four cases were cardiomyopathy secondary to iron overload in thalassemia patients.

We add T2*-weighted image in our protocol in examination of patient with thalassemia and we found that myocardial iron deposition was found on gradient-echo images, with lower signal at higher echo time (TE) values and this matched with study conducted by Cozma [22]. He reported that myocardial $\mathrm{T}^{*}$ was shown to be 
a more reliable indicator of true myocardial iron content as compared to serum ferritin levels or liver iron. Myocardial $\mathrm{T}^{*}<20 \mathrm{~ms}$ is significant iron deposition and $<10 \mathrm{~ms}$ is advanced iron deposition (Fig. 8).

In our study, we found that there was an agreement between echocardiography and cardiac MRI examination in evaluation of LVEF as demonstrated by Bland-Altman plot test Fig. 2. Among our 44 patients, the mean EF by echo was $54.4+19.7 \mathrm{SD}$ and by cardiac magnetic resonance was $47.2+21.6$ with a mean difference of $7.3 \%$. SD of bias was 9.7 and the $95 \%$ limits of agreement were 26.4 to 11.6. Our result are quite different from study conducted by Hoffmann et al. [23]. All of them found that mean differences between EF by cardiac magnetic resonance and echocardiography images were below $5 \%$; however, we found that mean difference was $7 \%$.

In our study, there was an overestimation of LVEF by echocardiography compared to CMR with a mean difference of about $7 \%$ between the two imaging modalities. As well, our results agreed with Rasha et al. [24] who observed agreement between echocardiography and CMR for estimation of LVEF in 37 patients with left ventricular dysfunction due to ischemic heart disease; the mean EF by CMR and echocardiography was $33.3 \%$ and $29 \%$ respectively with a mean difference of $4.3 \%$. SD of bias was 6.73 and the $95 \%$ limit of agreement was -8.90 to $7.5 \mathrm{~m}$ and our result were mean EF by echo was 54.4 and by cardiac magnetic resonance was 47.2 with a mean difference of $7.3 \%$. SD of bias was 9.7 and the $95 \%$ limits of agreement were -26.4 to 11.6 with higher values in our study.

We have relatively high percentage of bias in comparison to all previous reported study, as all these studies were in adults and our study was in children, and need for further agreement that MRI is more accurate than echo in functional cardiac assessment due to relatively different anatomy between adult and children and challenge of echo technique in children.

As regards the delayed contrast enhancement finding in our study, LGE is found in 15 cases out of 31 cases (48.4\%); however, the remaining 13 cases have no contrast study due to medical problems, the parent refusing or escape of contrast out of cannula. The enhancing cases were 7 DCM, 4 HCM, 1 ARVD, 1 infiltrative cardiomyopathy, and two ischemic cases. The following regional patterns are presented in Table 4: mid-wall $(n=$ 9), sub-endocardial extending to epicardial surface (transmural) $(n=4)$ (Fig. 3), and sub-epicardial $(n=2)$, sub-endocardial only $(n=2)$.

We found that there was correlation between the presence of LGE and MRI LVEF and LV volumes. The LVEF was lower and LV volume indices included (LVEDV, LVESV) were higher in patients with LGE compared with those without LGE with a statistically significant

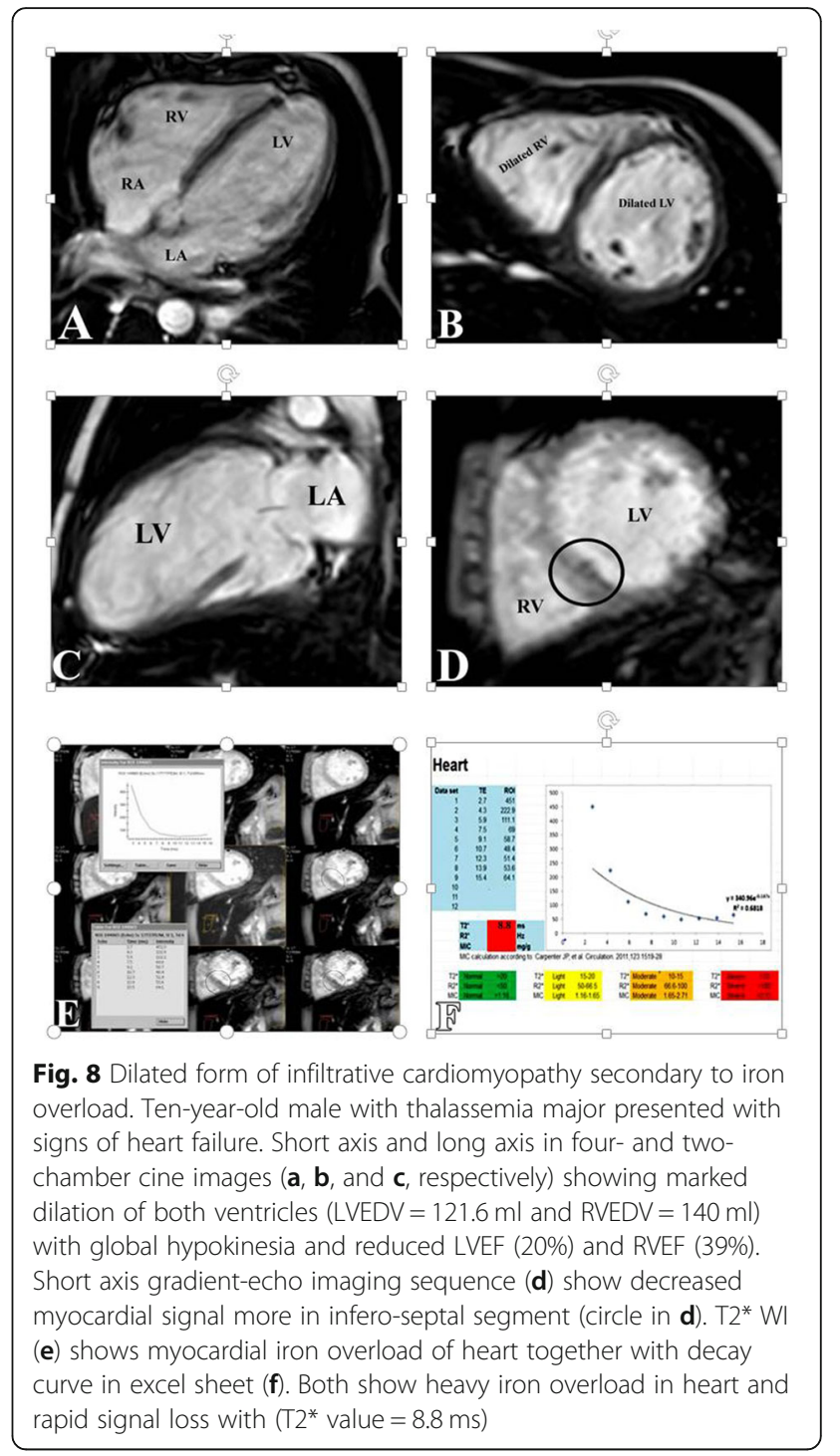

difference $(p$ value $=0.001, p$ value $=0.003$, and $p$ value $=$ 0.005 , respectively).

Also, a major adverse effect found in 14 of our cases with higher incidence in enhancement cases (80\%) as compared to non-enhancement ones $(20 \%)$ with a statistically significant difference ( $p$ value $\leq 0.001)$. So as regards the correlation between enhancement and other variables, we found that adverse cardiac prognosis is associated with the presence of LGE.

These results agree with adult study carried out by $\mathrm{Wu}$ et al. [25] that correlates the presence and extent of CMR LGE with a higher risk of adverse cardiac outcomes in patients with non-ischemic cardiomyopathy (NICM) and LVEF 35\% and LVEF trended lower and LV volume indexes were higher in those patients with LGE compared with those without LGE. And in correlation with the study also carried out by Assomull et al. [26]. 
Although we found a significantly higher incidence of adverse cardiac outcomes in NICM patients with positive LGE, the absence of LGE did not exclude the occurrence of adverse effect and we found an adverse effect in two patient (12.5\%) without enhancement and this is in agreement with study carried out by Wu et al. (25) that found adverse cardiac effect in three patients of her study cases (9\%) without LGE and they explained that not all adverse cardiac effect are caused by fibrosis, but may occur due to other causes such as by bundle branch or inter fasicular re-entry in arrhythmia and others.

From previous results LGE image can be used as clinical tool for suspecting cases with bad clinical out come and aid in management of these cases.

\section{Limitations}

The limitations were as follows:

- Compliance is essential for patients younger than 8 years old and some patients not sedated by oral chloral or refused by parents and may also need to IV sedative or general anesthesia which were problematic.

- Identification of late enhancement in children may be inadequate due to respiratory or motion artifacts caused by spontaneous breathing under sedation. Also, spatial resolution of the CMR sequence might be too low to identify small areas of local fibrosis in neonates and infants.

- We depend mainly in our study on correlation with echo finding, clinical data, and laboratory investigation as cardiac biopsy is not available.

\section{Conclusion}

Our study included 44 patients with different types of cardiomyopathy, in which, DCM represents the commonest type (43.2\%) followed by HCM (25\%), and restrictive cardiomyopathy was the one with low incidence (2.3). Also, other types as ARVD, LVNC, infiltrative, and chemotherapy-induced cardiomyopathy (CICM) were represented in variable small percentage.

In our study, there was agreement between both echo and MRI in evaluation of LVEF with echo showing overestimation and MRI was accurate and better evaluating tool. Also, MRI is the method of choice in right ventricular assessment as regards both morphological and functional parameters due to bad quality of image in echo by anterior position of RV behind sternum.

LGE was a common finding (48.4\%) and it showed different patterns that differentiate between ischemic and non-ischemic cardiomyopathy types. Also, in nonischemic types, different patterns of LGE was identified and is useful for detecting the underlying etiology.
We found that there was a correlation between the presence of LGE and left ventricle functional parameter in the form of lower LVEF and increased LVEDV, LVESV in patients with enhancement compared with those without with a statistically significant difference $(p$ value $=0.001, \quad p$ value $=0.003$, and $p$ value $=0.005$, respectively).

Furthermore, we also found that major adverse effect occurs with higher incidence in enhancement cases $(80 \%)$ as compared to non-enhancement $(20 \%)$ with a statistically significant difference $(p$ value $<0.001)$.

So, cardiac MRI can be considered an important noninvasive imaging modality not only for assessment but also in differentiation between ischemic and nonischemic cardiomyopathy in the pediatric age group. Using its different techniques allows a better assessment of morphologic and functional parameters in cardiomyopathy in children.

Moreover, the late gadolinium enhancement is considered a promising non-invasive tool in the detection and quantification of myocardial scars that are considered of high importance in diagnosis, categorization, and detection of etiology in most cases of different types of cardiomyopathy, in addition to risk stratification which can be an important step in patient management.
Abbreviations
ARVD/C: Arrhythmogenic right ventricular dysplasia/cardiomyopathy: BSA: Body surface area; CICM: Chemotherapy-induced cardiomyopathy; CMRI: Cardiovascular magnetic resonance imaging; COP: Cardiac output; DCM: Dilated cardiomyopathy; ECG: Electrocardiogram; EDV: End-diastolic volume; EF: Ejection fraction; ESV: End-systolic volume; HCM: Hypertrophic cardiomyopathy; HD: Heart disease; HLA: Horizontal long axis;
HOCM: Hypertrophic obstructive cardiomyopathy; IV: Intravenous; LA: Left atrium; LGE: Late gadolinium enhancement; LV: Left ventricle; LVNC: Left ventricular non-compaction; LVOT: Left ventricle outflow tract; MRI: Magnetic resonance imaging; NICM: Non-ischemic cardiomyopathy; PC: Phase contrast; RA: Right atrium; RC: Restrictive cardiomyopathy; ROI: Region of interest; RV: Right ventricle; RVOT: Right ventricle outflow tract; SA: Short axis; SAM: Systolic anterior motion; SD: Standard deviation; SI: Signal intensity; SSFP: Steady-state free procession; STIR: Saturated triple-inversion recovery; SV: Stroke volume; SWMA: Segmental wall motion abnormalities; TE: Echo time; VLA: Vertical long axis; WMA: Wall motion abnormalities

\begin{abstract}
Acknowledgements
First and foremost, praise to Allah for help, generosity, and giving me the force and confidence to finish this work. I would like to express my deep thanks and gratitude to Prof. Dr. Nevertiti Kamal El-Din Eid, Professor of Diagnostic Radiology, Faculty of Medicine, Mansoura University, for her great help, sincere effort, and valuable directions during the conduct of this work. I would like to express my sincere gratitude to the spirit of my father, my mother, my brothers, my sister, and all my family for helping me a lot during my life, supporting me, praying to me, and giving me the power to go on in my practical life. I wish to express my thanks and gratitude to my husband and my daughters, they bear and endure me a lot.
\end{abstract}

\section{Authors' contributions}

H.G.Abu-El Fadl analyzed and interpreted all patient data included in the study. H.G.Abu-EL Fadl, N.K. Eid were a major contributor in writing the manuscript. All authors read and approved the final manuscript. All authors made substantial contribution to conception, design, and statistical analysis of the study. All authors were actively involved in reviewing the manuscript. They all approved the final version of the manuscript. 


\section{Funding}

This study had no funding from any resource.

\section{Availability of data and materials}

The datasets used and/or analyzed during the current study are available from the corresponding author on reasonable request.

\section{Ethics approval and consent to participate}

This study was approved by the Research Ethics Committee of the Faculty of Medicine at Mansoura University in Egypt. All patients included in this research were under age of 18 years old, so written informed consent to publish the data contained within this study were given by their parents or legal guardians.

\section{Consent for publication}

Not applicable

\section{Competing interests}

The authors declare that they have no competing interests.

Received: 1 July 2019 Accepted: 30 August 2019

Published online: 30 October 2019

\section{References}

1. Gopalakrishnan, Vanathi, Prahlad G. Menon, and Shobhit Madan. (2014): "A novel framework to enhance scientific knowledge of cardiovascular MRI biomarkers and their application to pediatric cardiomyopathy classification." IWBBIO.

2. Behairy N.H., Mansour S.M (2014): Pattern of delayed myocardial enhancement: a key to differentiate ischemic and non-ischemic cardiomyopathies.1 (45):53-60.

3. Bogaert J, Taylor AM (2012) Cardiac anatomy. In: Bogaert J, Dymarkowski S, Taylor AM, Muthurangu V (eds) Clinical Cardiac MRI. Springer-Verlag Berlin Heidelberg, Germany, pp 69-91

4. Harris SR, Glockner J, Misselt AJ, Syed IS, Araoz PA (2008) Cardiac MR imaging of non-ischemic cardiomyopathies. MagnReson Imaging Clin N Am. 16:165-183

5. Nugent AW, Daubeney PE, Chondros P et al (2003) The epidemiology of childhood cardiomyopathy in Australia. N Engl J Med 348:1639-1646

6. Hong YM (2013) Cardiomyopathies in children. Korean J Pediatr 56:52-59

7. lacucci I, Carbone I, Cannavale G et al (2013) Myocardial oedema as the sole marker of acute injury in Takotsubo cardiomyopathy: a cardiovascular magnetic resonance (CMR) study. La Radiologia Medica 118(8):1309-1323

8. Latus H, Gummel K, Klingel K, Moysich A, Khalil M, Mazhari N et al (2015) Focal myocardial fibrosis assessed by late gadolinium enhancement cardiovascular magnetic resonance in children and adolescents with dilated cardiomyopathy. J Cardiovasc Magn Reson 17:34

9. Lehrke S, Lossnitzer D, Schöb M et al (2011) Use of cardiovascular magnetic resonance for risk stratification in chronic heart failure: prognostic value of late gadolinium enhancement in patients with non-ischaemic dilated cardiomyopathy. Heart 97(9):727-732

10. Rickers C, Wilke NM, Jerosch-Herold M et al (2005) Utility of cardiac magnetic resonance imaging in the diagnosis of hypertrophic cardiomyopathy. Circulation 112(6):855-861

11. Maron MS, Olivotto I, Harrigan C, Appelbaum E, Gibson CM, Lesser JR, Haas TS, Udelson JE, Manning WJ, Maron BJ (2011) Mitral valve abnormalities identified by cardiovascular magnetic resonance represent a primary phenotypic expression of hypertrophic cardiomyopathy. Circulation 124:40-47

12. Luckie M, Khattar RS (2008) Systolic anterior motion of the mitralvalve: beyond hypertrophic cardiomyopathy. Heart 94(11):1383-1385

13. Allen BD, Choudhury L, Barker AJ, van Ooij P, Collins JD, Bonow RO, Carr JC, Markl M. (2014): Three-dimensional haemodynamics in patients with obstructive and non-obstructive hypertrophic cardiomyopathy assessed by cardiac magnetic resonance. Eur Heart J Cardiovasc Imaging.

14. Maron MS, Maron BJ (2015) Clinical impact of contemporary cardiovascular magnetic resonance imaging in hypertrophic cardiomyopathy. Circulation 132(4):292-298

15. Maron BJ, Spirito P, Ackerman MJ, Casey SA, Semsarian C, Estes NM III et al (2013) Prevention of sudden cardiac death with implantable cardioverterdefibrillators in children and adolescents with hypertrophic cardiomyopathy. J Am Coll Cardiol 61(14):1527-1535
16. Moon JC, McKenna WJ (2012) Myocardial crypts: a prephenotypic marker of hypertrophic cardiomyopathy? Circ Cardiovasc Imaging 5(4):431-432

17. Mikolich JR, Lisko J, Boniface NC, Mikolich BM (2013) What is the role of cardiac MRI in the diagnosis of left ventricular non-compaction? J Cardiovasc Magn Reson 15(1):P157

18. Zuccarino F et al (2015) Left ventricular noncompaction: imaging findings and diagnostic criteria. Am J Roentgenol 204(5):519-530

19. Ichida F (2009) Left ventricular noncompaction. Circ J 73:19-26

20. Grosse-Wortmann L, Etoom Y, Govindapillai S, McCrindle B, Manlhiot C, Yoo SJ (2012) MRI in childhood arrhythmogenic right ventricular cardiomyopathy and proposed modification of the Task Force Criteria for children. J Cardiovasc Magn Reson 14(S1):01

21. Rammos, A., Meladinis, V., Vovas, G., \& Patsouras, D (2017): Restrictive cardiomyopathies: The importance of noninvasive cardiac imaging modalities in diagnosis an dtreatment -a systemic review. Radiology research and practice, 2017

22. Cozma ARI (2013) Role of cardiac imaging in early diagnosis of $\beta$ thalassemia major's iron overload cardiomyopathy

23. Hoffmann R, von Bardeleben S, Cate FT et al (2005) Assessment of systolic left ventricular function: a multi-centre comparison of cineventriculography, cardiac magnetic resonance imaging, unenhanced and contrast-enhanced echocardiography. Eur Heart J 2:607-616

24. Hussein RS, Ibrahim AS, El-Hameed AMA et al (2013) Does CMR have an additive role over echo in evaluating ischemic LV dysfunction? Egypt J Radiol Nud Med 44:475-482

25. Wu KC, Weiss RG, Thiemann DR, Kitagawa K, Schmidt A, Dalal D et al (2008) Late gadolinium enhancement by cardiovascular magnetic resonance heralds an adverse prognosis in non-ischemic cardiomyopathy. J Am Coll Cardiol 51(25):2414-2421

26. Assomull RG, Prasad SK, Lyne J et al (2006) Cardiovascular magnetic resonance, fibrosis, and prognosis in dilated cardiomyopathy. J Am Coll Cardiol 48(10):1977-1985

\section{Publisher's Note}

Springer Nature remains neutral with regard to jurisdictional claims in published maps and institutional affiliations.

\section{Submit your manuscript to a SpringerOpen ${ }^{\circ}$ journal and benefit from:}

- Convenient online submission

- Rigorous peer review

- Open access: articles freely available online

- High visibility within the field

- Retaining the copyright to your article

Submit your next manuscript at $>$ springeropen.com 